\title{
Hybrid Invariance in Bipedal Robots with Series Compliant Actuators
}

\author{
B. Morris* and J.W. Grizzle*
}

\begin{abstract}
Stable walking motions in bipedal robots can be modeled as asymptotically stable periodic orbits in nonlinear systems with impulse effects. The method of hybrid zero dynamics, previously used to analyze planar walking in bipeds with one degree of underactuation, is extended to address the increased degrees of underactuation and the additional impact invariance conditions that arise when actuator dynamics are explicitly modeled. The resultant controller is parameterized and includes a discrete feedback in the parameters that is active only in the instantaneous double support phase. The controller design method is illustrated on a five-link planar walker with series compliant actuation, that is, a robot where a compliant element has been deliberately inserted between each actuated joint and its corresponding motor in order to increase the overall energy efficiency of locomotion.
\end{abstract}

\section{INTRODUCTION}

In legged robots, the physical introduction of tuned springs into an otherwise rigid mechanism can significantly improve energy efficiency. The energetic benefits are twofold: within the strides of walking and running, springs can store and release some of the energy that would otherwise be lost as actuators do negative work [2]; and at foot touchdown events, springs isolate reflected motor inertias from the energydissipating effects of rigid collisions. These and other uses of flexible elements have been demonstrated on running robots such as RHex [20], Scout [16], Sprawlita [4], and the notably efficient ARL Monopod II [1]. And while the benefits of energy storage are most evident in running, in practice many robots must quite literally walk before they can run. In these cases compliance must be taken into account in the design and control of walking gaits, either explicitly by modeling, or implicitly by treating nonrigid effects as disturbances to a fully rigid model.

Obtaining the energetic benefits of compliance is not without cost: delivering torque through compliant elements poses several challenges for control design. There is an obvious increase in the degrees of freedom of the robot model, and hence, the degree of underactuation. This is a widely recognized issue in robotics; see [22], [21], [3] and references therein. An additional challenge particular to legged robots arises from the impulsive effects occurring when the swing leg impacts the ground. When torque at a joint is generated by a motor and drivetrain in series with a spring (as in this paper) the spring isolates the motor and drivetrain from the effects of an impact. Post-impact values of rotor position and velocity match their pre-impact

\footnotetext{
* This work was supported by NSF grant ECS-0600869. B. Morris and J.W. Grizzle are with the Control Systems Laboratory, EECS Department, University of Michigan, Ann Arbor, Michigan 48109-2122, USA. E-mail: \{morrisbj, grizzle\}@umich.edu.
}
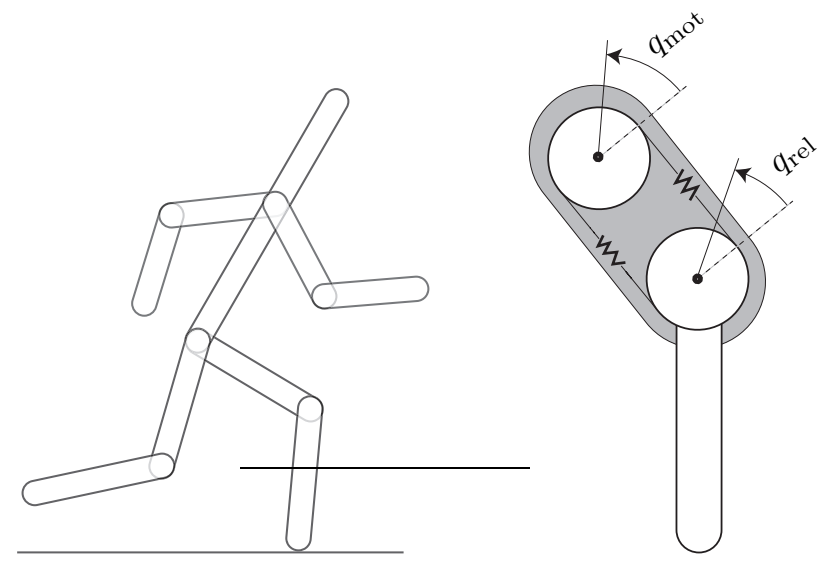

Fig. 1. Left: A representative example, intentionally anthropomorphic, of the class of $N$-link biped robot models considered. Right: A schematic of a rotational joint with series compliant actuation.

values, and similar boundary conditions arise for joint torque. Seemingly benign, these additional post-impact boundary conditions alter the structure of the impact map and can significantly complicate controller design.

The method of hybrid zero dynamics, as presented in [25] for the control of planar walking, assumed that any actuator dynamics were sufficiently fast that they could be neglected in the controller design process. The novel element of this paper is the extension of the hybrid zero dynamics framework to address unique aspects of stabilizing walking motions using actuators with nontrivial series compliance. Specific attention is given to the post-impact boundary conditions, which no longer satisfy the linear structures assumed in [25]. Treating actuator dynamics in this framework will lead to reduced dimensionality stability tests for closed-loop walking gaits despite the increased degrees of underactuation that accompany compliant actuation.

See Fig. 1 for a description of the class of robots considered in this paper, along with a schematic diagram of a flexible actuator. One example of the pictured compliant mechanism is the MIT Series Elastic Actuator, which uses stiff springs and an inner-loop feedback controller to achieve reliable force control [18], [17]. Another, the AMASC (Actuator with Mechanically Adjustable Series Compliance) designed by Hurst [11], consists of a drive motor connected in series with a pair of large, variable stiffness springs. Unlike the MIT Series Elastic Actuator, the AMASC is designed to mechanically store significant amounts of energy that would otherwise be wasted when the actuator does negative work.

The rest of the paper is organized as follows: Section II summarizes background material on systems with impulse effects, periodic orbits in such systems, and the method of Poincaré sections. Section III contains two theorems, the 
main theoretical results of the paper. The concept of a hybrid zero dynamics (HZD) is defined in a more general manner than in [25], followed by a theorem that provides sufficient conditions - and a controller design recipe-for stabilizing a periodic orbit in a nonlinear system with impulse effects. One of the conditions of this theorem, that the continuous-phase zero dynamics manifold is invariant under the impact map, can be difficult to meet in practical examples. The second theorem of this section provides conditions for constructing an embedding of the original system with impulse effects into a larger system with impulse effects where the mentioned impact invariance condition is easier to meet. Moving toward applications, Section IV presents a model of a class of planar biped robots with series actuation compliance; properties of the models are summarized that aid in applying the theorems of Section III. Section V presents the application of the results of Section III to a particular instance of the biped models of Section IV. A simulation of the closed-loop walking gait shows properties that are predicted by theory. Conclusions are given in Section VI.

\section{TeChnicAl BACKGRound}

\section{A. Systems with impulse effects}

Consider a control system

$$
\dot{x}=f(x)+g(x) u,
$$

where $x \in \mathcal{X}$, an open connected subset of $\mathbb{R}^{n}, u \in \mathbb{R}^{m}$, and $f$ and the columns of $g$ are $C^{\infty}$ vector fields on $\mathcal{X}$. An impact (or switching) surface is a co-dimension one $C^{\infty}$ submanifold $\mathcal{S}:=\{x \in \mathcal{X} \mid H(x)=0\}$, where $H: \mathcal{X} \rightarrow \mathbb{R}$ is $C^{\infty}$ and $\forall x \in \mathcal{S}, \frac{\partial H}{\partial x}(x) \neq 0$. An impact (or reset) map is a $C^{\infty}$ function $\Delta: \mathcal{S} \rightarrow \mathcal{X}$. A $C^{\infty}$ system with impulse effects is a model of the form

$$
\Sigma:\left\{\begin{aligned}
\dot{x} & =f(x)+g(x) u & & x^{-} \notin \mathcal{S} \\
x^{+} & =\Delta\left(x^{-}\right) & & x^{-} \in \mathcal{S},
\end{aligned}\right.
$$

where $x^{-}(t):=\lim _{\tau \nearrow t} x(\tau)$ and $x^{+}(t):=\lim _{\tau \searrow t} x(\tau)$ denote, respectively, the left and right limits of a trajectory, $x(t)$. In simple words, a trajectory of the model is specified by the differential equation (1) until its state "impacts" the hypersurface $\mathcal{S}$. At this point, the impact map $\Delta$ assigns a new initial condition from which the differential equation evolves until the next impact with $\mathcal{S}$. In order to avoid the state having to take on two values at the same instant, the impact event is, roughly speaking, described in terms of the values of the state "just prior to impact" at time " $t^{-}$", and "just after impact" at time " $t$ ". These values are represented by the left and right limits, $x^{-}$and $x^{+}$, respectively. A formal definition of a solution is easily written down by piecing together appropriately initialized solutions of (1); see [26], [8], [15], [5]. A choice must be made whether to take a solution $\varphi(t)$ of (2) to be a left- or a right-continuous function of time at each impact event; here, solutions are assumed to be right continuous as in [8].

\section{B. Periodic orbits}

A $C^{\infty}$ autonomous system with impulse effects is given by (2) with $u=0$, namely,

$$
\Sigma:\left\{\begin{array}{rlrl}
\dot{x} & =f(x) & & x^{-} \notin \mathcal{S} \\
x^{+} & =\Delta\left(x^{-}\right) & x^{-} \in \mathcal{S} .
\end{array}\right.
$$

A solution $\varphi(t)$ of (3) is periodic if there exists a finite $T>0$ such that $\varphi(t+T)=\varphi(t)$ for all $t \in\left[t_{0}, \infty\right)$. A set $\mathcal{O} \subset \mathcal{X}$ is a periodic orbit if $\mathcal{O}=\left\{\varphi(t) \mid t \geq t_{0}\right\}$ for some periodic solution $\varphi(t)$. While a system with impulse effects may certainly have periodic solutions that do not involve impact events, they are not of interest here because they could be studied more simply as solutions of (1). If a periodic solution has an impact event, then the corresponding periodic orbit $\mathcal{O}$ is not closed; see [8], [14]. Let $\overline{\mathcal{O}}$ denote its set closure. A periodic orbit $\mathcal{O}$ is transversal to $\mathcal{S}$ if its closure intersects $\mathcal{S}$ in exactly one point, and for $x^{*}:=$ $\overline{\mathcal{O}} \cap \mathcal{S}, L_{f} H\left(x^{*}\right):=\frac{\partial H}{\partial x}\left(x^{*}\right) f\left(x^{*}\right) \neq 0$ (in words, at the intersection, $\overline{\mathcal{O}}$ is not tangent to $\mathcal{S}$ ). Notions of stability in the sense of Lyapunov, asymptotic stability, and exponential stability of orbits follow the standard definitions; see [13, pp. 329], [8], [15].

\section{Poincaré return map}

The method of Poincaré sections is the primary tool used to test the stability of periodic orbits in nonlinear systems. When such orbits occur in systems with impulse effects, it is natural to select the impact surface $\mathcal{S}$ as the Poincaré section. To define the return map, let $\varphi\left(t, x_{0}\right)$ denote the maximal solution of $\dot{x}=f(x)$ in (3) with initial condition $x_{0}$ at time $t_{0}=0$. The time-to-impact function, $T_{I}: \mathcal{X} \rightarrow \mathbb{R} \cup\{\infty\}$, is defined by

$$
T_{I}\left(x_{0}\right):= \begin{cases}\inf \left\{t \geq 0 \mid \varphi\left(t, x_{0}\right) \in \mathcal{S}\right\} & \text { if } \exists t \text { such that } \\ & \varphi\left(t, x_{0}\right) \in \mathcal{S} \\ \infty & \text { otherwise. }\end{cases}
$$

The Poincaré return map, $P: \mathcal{S} \rightarrow \mathcal{S}$, is then given as (the partial map)

$$
P(x):=\varphi\left(T_{I} \circ \Delta(x), \Delta(x)\right) .
$$

The method of Poincaré sections can then be stated as follows:

Theorem 0: (Method of Poincaré Sections [14], [8], [15]) If the $C^{\infty}$ system with impulse effects (3) has a periodic orbit $\mathcal{O}$ with $x^{*}:=\overline{\mathcal{O}} \cap \mathcal{S}$ a singleton and $L_{f} H\left(x^{*}\right) \neq 0$, then the following are equivalent:

i) $x^{*}$ is an exponentially stable (resp., asymp. stable, or stable i.s.L.) fixed point of $P$;

ii) $\mathcal{O}$ is an exponentially stable (resp., asymp. stable, or stable i.s.L.) periodic orbit of $\Sigma$. 


\section{NEW RESUlts ON HYBRID ZERO DYNAMICS}

The paper [25] introduced the notion of a hybrid zero dynamics (HZD) for systems with impulse effects. The HZD is based on two principles that are ubiquitous in non-hybrid systems, namely invariance and attractivity. Many problems involving the existence and stability of periodic orbits in systems with impulse effects can be simplified if the system possesses an appropriately defined hybrid invariant manifold. In the context of planar bipedal locomotion, insight can be gained by studying the system restricted to the invariant submanifold, that is, the HZD.

The current paper presents two theorems that directly extend the results of [25], [14], and several others. In the first theorem, using [14], the HZD is extended to outputs with uniform vector relative degree greater than or equal to one. Previous work of [25] applies to outputs of uniform vector relative degree two (each output component has relative degree two and the associated decoupling matrix is square and invertible [12]). The key contribution of the second theorem is a novel dynamic controller that is active only at the impacts, whose function is to relax the conditions for achieving impact invariance when constructing an HZD.

\section{A. Hybrid Zero Dynamics for Systems with Uniform Vector} Relative Degree $k \geq 1$

The basic idea of an HZD is that an output should give rise to a zero dynamics for the continuous portion of the model [12] and the resulting zero dynamics manifold should be invariant under the impact map. This is formalized as follows:

Associate a $C^{\infty}$ output

$$
y=h(x)
$$

to (2) and assume that $h$ has vector relative degree $\left(k_{1}, \cdots, k_{m}\right)$ with respect to the continuous portion of the hybrid model (that is, $h_{i}$ has relative degree $k_{i}$ with respect to (1) and the decoupling matrix is square and invertible [12]) and that there exists $x_{0} \in \mathcal{X}$ such that $h\left(x_{0}\right)=0$. Let $\mathcal{Z}$ be the zero dynamics manifold for the continuous portion of the dynamics and let $u^{*}$ be the feedback (unique on $\mathcal{Z}$ ) such that for all $x \in \mathcal{Z}, f^{*}(x):=f(x)+g(x) u^{*}(x) \in T_{x} \mathcal{Z}$. If in addition, $\mathcal{S} \cap \mathcal{Z}$ is a $C^{\infty}$ manifold of dimension one less than $\mathcal{Z}$ and

$$
\Delta(\mathcal{S} \cap \mathcal{Z}) \subset \mathcal{Z}
$$

then $\mathcal{Z}$ is a hybrid zero dynamics manifold for $\Sigma$ and the restriction dynamics

$$
\left.\Sigma\right|_{\mathcal{Z}}:\left\{\begin{array}{clrl}
\dot{z} & =\left.f^{*}\right|_{\mathcal{Z}}(z) & z^{-} \notin \mathcal{S} \cap \mathcal{Z} \\
z^{+} & =\left.\Delta\right|_{\mathcal{S} \cap \mathcal{Z}}\left(z^{-}\right) & z^{-} \in \mathcal{S} \cap \mathcal{Z},
\end{array}\right.
$$

is called the hybrid zero dynamics of $\Sigma$, where $\left.f^{*}\right|_{\mathcal{Z}}$ and $\left.\Delta\right|_{\mathcal{S} \cap \mathcal{Z}}$ are the restrictions of $f^{*}$ and $\Delta$ to $\mathcal{Z}$ and $\mathcal{S} \cap \mathcal{Z}$, respectively.

For notational expediency, we assume in the following that the relative degree is the same for each output component. This level of generality is all that will be needed in the remainder of the paper. The developed results can be extended to systems with general vector relative degree, or to systems for which a vector relative degree is achievable by dynamic feedback; see [12].

Remark 1: When a system with impulse effects (2) has an output $h(x)$ with uniform vector relative degree $k$, the following are equivalent:

a) $\Delta(\mathcal{S} \cap \mathcal{Z}) \subset \mathcal{Z}$

b) $\forall x \in \Delta(\mathcal{S} \cap \mathcal{Z})$ and $\forall 0 \leq i \leq k-1 L_{f}^{i} h(x)=0$.

The following theorem gives HZD-based sufficient conditions for stabilization of a periodic orbit in an open-loop system with impulse effects.

Theorem 1: Consider a $C^{\infty}$ system with impulse effects (2) with a $C^{\infty}$ output (6) and hybrid zero dynamics (8). Suppose that (8) contains a periodic orbit $\mathcal{O}$ that is exponentially stable and transversal to $\mathcal{S}$. If in addition

1) the output $h$ has uniform vector relative degree $k$; and

2) there exists a vector of functions $\phi: \mathcal{X} \rightarrow \mathbb{R}^{n-m k}$ such that $L_{g} \phi \equiv 0$; and

$$
\Phi(x)=\left(\phi(x) ; h(x) ; L_{f} h(x) ; \cdots ; L_{f}^{k-1} h(x)\right)
$$

is a diffeomorphism on $\mathcal{X}$,

then the orbit $\mathcal{O}$ is exponentially stabilizable. For any choice of matrices $K_{0}, K_{1}, \cdots, K_{k-1}$ satisfying that $s^{k}+K_{k-1} s^{k-1}+\cdots K_{0}$ is Hurwitz, for $\epsilon>0$ sufficiently small, the feedback

$$
u(x)=\left(L_{g} L_{f}^{k-1} h(x)\right)^{-1}\left(\begin{array}{c}
-L_{f}^{k} h(x)+\ldots \\
\sum_{i=0}^{k-1} \frac{1}{\epsilon^{k-1}} K_{i} L_{f}^{i} h(x)
\end{array}\right),
$$

applied to (2) renders $\mathcal{O}$ exponentially stable in the (fulldimensional) closed-loop system

$$
\Sigma:\left\{\begin{aligned}
\dot{x} & =f(x)+g(x) u(x) & & x^{-} \notin \mathcal{S} \\
x^{+} & =\Delta\left(x^{-}\right) & & x^{-} \in \mathcal{S} .
\end{aligned}\right.
$$

Proof: As in [12, Prop. 6.1.5], introduce the coordinates $z=\phi(x)$ and $\eta=\left(h(x) ; \cdots ; L_{f}^{k-1} h(x)\right)$. In $(z ; \eta)$, the closed-loop dynamics $\dot{x}=f(x)+g(x) u(x)$ satisfies all of the hypotheses of [14, Thm. 2]. Hence, for $\epsilon$ sufficiently small, exponential stability of the orbit in the closed-loop system (11) is equivalent to exponential stability of the orbit in the zero dynamics (8).

\section{B. Using a Deadbeat Hybrid Extension to Achieve Impact Invariance}

One critical aspect of applying Theorem 1 in the context of bipedal locomotion is the selection of an output $h(x)$ that leads to a hybrid zero dynamics. Appropriately choosing an output so that $\mathcal{Z}$ is impact invariant is a nontrivial task, in general. In previous work, [25, Sec. V, Thm. 4] identified a class of holonomic ${ }^{1}$, uniform vector relative degree 2 outputs for which it is straightforward to meet the impact invariance condition. The reasoning employed in [25] relied heavily

\footnotetext{
${ }^{1}$ The output function $h$ depended only on the configuration variables of the robot, hence the terminology "holonomic".
} 
on the fact that both the impact map $\Delta$ and $L_{f} h$ were linear in the generalized velocity coordinate. This linearity property breaks down already for $L_{f}^{2} h$ (equivalently, for outputs with uniform vector relative degree $\geq 3$ ), and in the context of compliant actuation, the impact map $\Delta$ is affine in the generalized velocity coordinate (not strictly linear). Without linearity, giving checkable conditions for impact invariance becomes very hard. We will overcome this obstacle by embedding the system with impulse effects (2) in a larger, parameterized system called a deadbeat hybrid extension. With the introduction of parameterized outputs, a discrete feedback element becomes available-the parameter update law.

The following theorem illustrates the use of a parameter update law in achieving a hybrid zero dynamics. Under the given hypothesis, the extra dimensionality associated with the parameters does not significantly complicate the Poincaré return map.

Theorem 2: Consider a $C^{\infty}$ system with impulse effects

$$
\Sigma:\left\{\begin{aligned}
\dot{x} & =f(x)+g(x) u & & x^{-} \notin \mathcal{S} \\
x^{+} & =\Delta\left(x^{-}\right) & & x^{-} \in \mathcal{S},
\end{aligned}\right.
$$

with an $n$-dimensional state manifold $\mathcal{X}$ and $m$-dimensional inputs $u$. Let $\mathcal{A}$ be an open subset of $\mathbb{R}^{p}$, for some $p \geq 1$, and let $h: \mathcal{X} \times \mathcal{A} \rightarrow \mathbb{R}^{m}$ be $C^{\infty}$.

Suppose furthermore that

1) $\forall \alpha \in \mathcal{A}$,

$$
y=h(x, \alpha),
$$

has uniform vector relative degree $k$;

2) there exists a non-empty $C^{\infty}$ submanifold $\mathcal{Z}$ such that $\forall \alpha \in \mathcal{A}$, $\mathcal{Z}_{\alpha}:=\left\{x \in \mathcal{X} \mid h(x, \alpha)=0, \cdots, L_{f}^{k-1} h(x, \alpha)=0\right\}$ is diffeomorphic to $\mathcal{Z}$;

3) $\mathcal{S} \cap \mathcal{Z}_{\alpha}$ is independent of $\alpha$; denote it by $\mathcal{S} \cap \mathcal{Z}_{\diamond}$;

4) $\mathcal{S} \cap \mathcal{Z}_{\diamond}$ is $C^{\infty}$ and has dimension one less than $\mathcal{Z}$; and

5) there exits a $C^{\infty}$ function $\Delta_{1}: \mathcal{S} \rightarrow \mathcal{A}$ such that, $\forall x \in \mathcal{S} \cap \mathcal{Z}_{\diamond}$, the values $\xi=\Delta(x), \alpha=\Delta_{1}(x)$ result in

$$
h(\xi, \alpha)=0, \cdots, L_{f}^{k-1} h(\xi, \alpha)=0 .
$$

Then the control system,

$$
\tilde{\Sigma}:\left\{\begin{aligned}
{\left[\begin{array}{c}
\dot{x} \\
\dot{\alpha}
\end{array}\right] } & =\left[\begin{array}{c}
f(x)+g(x) u \\
0
\end{array}\right] x^{-} \notin \mathcal{S} \\
{\left[\begin{array}{l}
x^{+} \\
\alpha^{+}
\end{array}\right] } & =\left[\begin{array}{c}
\Delta\left(x^{-}\right) \\
\Delta_{1}\left(x^{-}\right)
\end{array}\right] \quad x^{-} \in \mathcal{S}
\end{aligned}\right.
$$

with output

$$
y=h(x, \alpha)
$$

has a hybrid zero dynamics. Moreover,

a) the hybrid zero dynamics manifold of (14) is $\mathcal{Z}_{\mathcal{A}}:=\cup_{\alpha \in \mathcal{A}}\left(\mathcal{Z}_{\alpha}, \alpha\right)$

b) $\mathcal{Z}_{\mathcal{A}} \cap(\mathcal{S} \times \mathcal{A})=\left(\mathcal{S} \cap \mathcal{Z}_{\diamond}\right) \times \mathcal{A}$, and c) the Poincare map of the hybrid zero dynamics, $P_{\text {zero }}: \mathcal{S} \cap \mathcal{Z}_{\diamond} \times \mathcal{A} \rightarrow \mathcal{S} \cap \mathcal{Z}_{\diamond} \times \mathcal{A}$, has the form

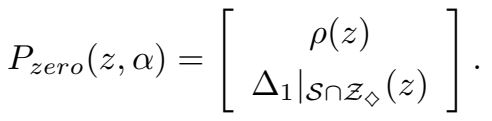

Proof: Hypotheses 1) and 2) imply immediately two things: that $\forall \alpha \in \mathcal{A}$ the continuous part of (12) with output (13) has a well-defined, zero dynamics manifold ${ }^{2}$ $\mathcal{Z}_{\alpha}$, and that the continuous portion of (14) with output (13) has a well-defined zero dynamics manifold, denoted temporarily by $\tilde{\mathcal{Z}}$. Again using Hypothesis 2 ), it follows that $\tilde{\mathcal{Z}}=\cup_{\alpha \in \mathcal{A}}\left(\mathcal{Z}_{\alpha}, \alpha\right)$, and hence the set $\tilde{\mathcal{Z}}=\mathcal{Z}_{\mathcal{A}}$ is a zero dynamics manifold of the continuous portion of (14). Next, note that by Hypothesis 3),

$$
\begin{aligned}
\mathcal{Z}_{\mathcal{A}} \cap(\mathcal{S} \times \mathcal{A}) & =\left(\cup_{\alpha \in \mathcal{A}}\left(\mathcal{Z}_{\alpha}, \alpha\right)\right) \cap\left(\cup_{\alpha \in \mathcal{A}}(\mathcal{S}, \alpha)\right) \\
& =\cup_{\alpha \in \mathcal{A}}\left(\mathcal{S} \cap \mathcal{Z}_{\alpha}, \alpha\right) \\
& =\cup_{\alpha \in \mathcal{A}}\left(\mathcal{S} \cap \mathcal{Z}_{\diamond}, \alpha\right) \\
& =\left(\mathcal{S} \cap \mathcal{Z}_{\diamond}\right) \times \mathcal{A},
\end{aligned}
$$

establishing part $\mathrm{b}$ ) of the Theorem. This and Hypothesis 4) imply that $\mathcal{Z}_{\mathcal{A}} \cap(\mathcal{S} \times \mathcal{A})$ is a $C^{\infty}$ submanifold of $\mathcal{X} \times \mathcal{A}$, and has dimension one less than $\mathcal{Z}_{\mathcal{A}}$. By Hypothesis 5) and Remark 1, $\mathcal{Z}_{\mathcal{A}} \cap(\mathcal{S} \times \mathcal{A})$ is invariant under the impact map of (14). It follows that (14) has an HZD with zero dynamics manifold $\mathcal{Z}_{\mathcal{A}}$, proving part a) of the Theorem. The corresponding restriction dynamics of (14) is

$$
\left.\tilde{\Sigma}\right|_{\mathcal{Z}_{\mathcal{A}}}:\left\{\begin{array}{l}
{\left[\begin{array}{c}
\dot{z} \\
\dot{\alpha}
\end{array}\right]=\left[\begin{array}{c}
\left.f^{*}\right|_{\mathcal{Z}_{\alpha}}(z, \alpha) \\
0
\end{array}\right] \quad z^{-} \notin \mathcal{S} \cap \mathcal{Z}_{\diamond}} \\
{\left[\begin{array}{c}
z^{+} \\
\alpha^{+}
\end{array}\right]=\left[\begin{array}{c}
\left.\Delta\right|_{\mathcal{S} \cap \mathcal{Z}_{\diamond}}\left(z^{-}\right) \\
\left.\Delta_{1}\right|_{\mathcal{S} \cap \mathcal{Z}_{\diamond}}\left(z^{-}\right)
\end{array}\right] \quad z^{-} \in \mathcal{S} \cap \mathcal{Z}_{\diamond},}
\end{array}\right.
$$

from which the form of the Poincaré map is immediate, thus proving part c).

\section{Remarks}

If a nonlinear system with impulse effects has an HZD, Theorem 1 provides sufficient conditions for being able to exponentially stabilize a periodic orbit, such as a walking motion of a bipedal robot. As in [25, Sec. VI], one interesting implication of Theorem 1 is the possibility of creating the periodic orbit on the basis of the HZD, which results in a lower-dimensional design problem.

Theorem 2 provides additional flexibility in meeting the hybrid invariance condition (7) by embedding the hybrid system in a special kind of dynamic extension. The dynamic extension is deadbeat in that the additional states are updated only at impacts, and their new values depend only on the original states of the system. This is beneficial because then the existence and stability of a fixed point of the Poincaré map of the restriction dynamics, given by (15), is determined completely by $\rho$; the term $\Delta_{1}$ is deadbeat. In other words, the deadbeat hybrid extension has not significantly complicated

\footnotetext{
${ }^{2}$ This does not imply that there exists a value of $\alpha$ for which $\mathcal{Z}_{\alpha}$ is a hybrid zero dynamics manifold of (12). No such value for $\alpha$ need exist.
} 
the determination of the stability properties of a periodic orbit on the basis of the HZD.

In (14), the switching surface is $\mathcal{S} \times \mathcal{A}$. It has been written in the given form to emphasize that the switching condition does not depend the value of $\alpha$.

\section{Application to Planar Bipedal Walkers}

This section provides models for a class of planar bipedal walkers, with and without actuator dynamics. Key properties of the models that are useful for checking the hypotheses of Theorems 1 and 2 are then succinctly summarized. These properties take advantage of the fact that the hypotheses of Theorems 1 and 2 are invariant under regular static state feedback (applied to the continuous portion of the model) and coordinate changes.

\section{A. Robot model without actuator dynamics}

As in [25, Sec. II], consider a bipedal robot consisting of $N$ links connected in a planar tree structure to form two identical legs with knees, but without feet, with the legs connected at a common point called the hips, and possibly other limbs (such as a torso, etc.). All links have mass, are rigid, and are connected in revolute joints (see Fig. 2). It is assumed that no actuation is applied between the stance leg and the ground, while all other joints are independently actuated, and hence there are $(N-1)$ controls. As shown in [6], [7], addressing the control of robots without actuation between the stance leg and ground is an important step in achieving anthropomorphic walking motions in robots with non-trivial feet and actuated ankles. Further details on the model are given in [25, Sec. II], along with assumptions on the walking gait (instantaneous double support phase, no slipping nor rebound at impact, motion from left to right, symmetric gait). A rigid impact is used to model the contact of the swing leg with the ground.

The configuration coordinates of the robot in single support (also called the stance phase) are denoted by $q=\left(q_{1} ; \cdots ; q_{N}\right) \in \mathcal{Q}$, the state space is denoted by $T \mathcal{Q}$. The method of Lagrange leads to the mechanical model

$$
D(q) \ddot{q}+C(q, \dot{q}) \dot{q}+G(q)=B u,
$$

where $B$ is an $N \times(N-1)$ constant matrix with rank $(N-1)$. Letting $x:=(q ; \dot{q})$, and defining $f$ and $g$ in the obvious manner, the mechanical model is expressed in state variable form as

$$
\dot{x}=f(x)+g(x) u .
$$

The hybrid model of the robot (single support phase Lagrangian dynamics plus impact map) is constructed by specifying the impact or switching surface

$\mathcal{S}=\left\{(q ; \dot{q}) \in T \mathcal{Q} \mid \mathbf{y}_{2}(q)-\mathbf{y}_{1}(q)=0, \mathbf{x}_{2}(q)-\mathbf{x}_{1}(q)>0\right\}$,

to be the set of points where the swing leg height is zero and positioned in front of the stance leg. The impact map $\Delta: \mathcal{S} \rightarrow T \mathcal{Q}$ is computed as in [10], [8]. When the swing leg contacts the ground, a rigid collision gives rise to a jump in the velocity coordinates. So that the same mechanical model

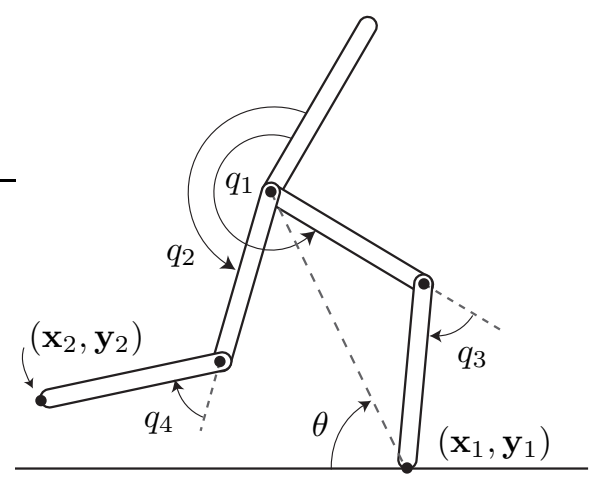

Fig. 2. A coordinate diagram of the robot model used in this case study. Angles are positive in the counterclockwise direction. All links have length $0.5 \mathrm{~m}$. The thigh and shin links have masses of 0.5 and $0.75 \mathrm{~kg}$ respectively. The torso link has a mass of $27.5 \mathrm{~kg}$. Mass distributions are uniform. The motors' reflected inertias are $0.404 \mathrm{~kg} \cdot \mathrm{m}^{2}$ at each actuated joint. Each motor acts through a series spring having an effective stiffness of $550 \mathrm{Nm} / \mathrm{rad}$.

can be used independent of which leg is the stance leg, the coordinates must also be relabeled at impact, giving rise to a jump in the configuration variables as well; see[8], [25]. The corresponding system with impulse effects is written as

$$
\Sigma:\left\{\begin{aligned}
\dot{x} & =f(x)+g(x) u & & x^{-} \notin \mathcal{S} \\
x^{+} & =\Delta\left(x^{-}\right) & & x^{-} \in \mathcal{S} .
\end{aligned}\right.
$$

\section{B. Robot model with compliant actuation}

Assume now that the vector of torques applied to the robot model (17) is generated through a compliant model of the form

$$
\begin{aligned}
u & =k\left(q_{m}-q_{a}\right) \\
J \ddot{q}_{m}+k\left(q_{m}-q_{a}\right) & =u_{m},
\end{aligned}
$$

where $q_{m} \in \mathcal{Q}_{m}$ is an $(N-1)$ tuple of motor angles, $q_{a}$ is a vector of the relative angles corresponding to the $(N-1)$ actuated joints of the robot, $u_{m}$ is the vector of $(N-1)$ motor torques, $k$ is a diagonal matrix of (positive) spring constants and $J$ is a diagonal matrix of (positive) rotor inertias. The stance phase dynamics of the robot is now given by the $(2 N-1)$ DOF Lagrangian system

$$
\begin{aligned}
D(q) \ddot{q}+C(q, \dot{q}) \dot{q}+G(q) & =B k\left(q_{m}-q_{a}\right) \\
J \ddot{q}_{m}+k\left(q_{m}-q_{a}\right) & =u_{m} .
\end{aligned}
$$

Letting $x_{m}:=\left(q_{m} ; \dot{q}_{m}\right)$ and $x_{e}:=\left(x ; x_{m}\right)$, this is easily expressed as

$$
\dot{x}_{e}=f_{e}\left(x_{e}\right)+g_{e}\left(x_{e}\right) u_{m} .
$$

The corresponding model with impulse effects is written as

$$
\Sigma_{e}: \begin{cases}\dot{x}_{e}=f_{e}\left(x_{e}\right)+g_{e}\left(x_{e}\right) u_{m} & x_{e}^{-} \notin \mathcal{S}_{e} \\ x_{e}^{+}=\Delta_{e}\left(x_{e}^{-}\right) & x_{e}^{-} \in \mathcal{S}_{e}\end{cases}
$$

where the state manifold is $\mathcal{X}_{e}=T \mathcal{Q} \times T \mathcal{Q}_{m}$ and because the impact condition depends only on the state of the biped 
(and not on the motor angles) $\mathcal{S}_{e}=\mathcal{S} \times T \mathcal{Q}_{m}$. Following [10], [8], the impact map $\Delta_{e}: \mathcal{S}_{e} \rightarrow \mathcal{X}_{e}$ has the form

$$
\Delta_{e}\left(x_{e}^{-}\right)=\left[\begin{array}{l}
\Delta\left(x^{-}\right) \\
\Delta_{m}\left(x^{-}, x_{m}^{-}\right)
\end{array}\right]
$$

where $\Delta$ is as in (19) and $\Delta_{m}$ imposes continuity ${ }^{3}$ in the motor positions and velocities across the impact. Hence, the only "jumps" in the motor variables $x_{m}$ are those due to leg swapping (recall that the robot coordinates are permuted at impact to reflect leg swapping).

\section{Model Properties}

Some properties of the mechanical models (17) and (22) are now summarized. These properties provide information on the zero dynamics of (18) and (23), and hence useful information on the HZD of (24) in light of applying Theorem 2 to the biped model with actuator dynamics. In the following, we choose configuration coordinates for (17) as $q=\left(q_{a} ; \theta\right)$, where $\theta$ references a position on the robot to the world frame (see Fig 2).

Proposition 1: Let $\sigma$ be the angular momentum of the biped about the contact point of the support leg with the ground. In the coordinates, $q=\left(q_{a} ; \theta\right)$,

a) the inertia matrix $D$ of (17) is independent of $\theta$;

b) (18) is globally feedback equivalent to

$$
\begin{aligned}
\dot{\sigma} & =-\frac{\partial V}{\partial \theta}(q) \\
\dot{\theta} & =\frac{\sigma}{d_{N, N}\left(q_{a}\right)}+R\left(q_{a}\right) \dot{q}_{a} \\
\ddot{q}_{a} & =v,
\end{aligned}
$$

where $V$ is the potential energy of the robot model (17),

$$
R\left(q_{a}\right)=-\left[\frac{d_{N, 1}\left(q_{a}\right)}{d_{N, N}\left(q_{a}\right)}, \cdots, \frac{d_{N, N-1}\left(q_{a}\right)}{d_{N, N}\left(q_{a}\right)}\right]
$$

and $d_{i, j}$ are the elements of $D$.

The proof and the required feedback are given in [9], and are based on [23], [19]. Because the actuator dynamics (20) is globally feedback equivalent to a vector of double integrators, the next result follows from basic results in [12] dealing with dynamic extensions.

Proposition 2: Let $q=\left(q_{a} ; \theta\right)$ and let $\sigma$ be the angular momentum of the biped about the contact point of the support leg with the ground. Then (23) is globally feedback equivalent to

$$
\begin{aligned}
\dot{\sigma} & =-\frac{\partial V}{\partial \theta}(q) \\
\dot{\theta} & =\frac{\sigma}{d_{N N}\left(q_{a}\right)}+R\left(q_{a}\right) \dot{q}_{a} \\
\ddot{q}_{a} & =v \\
\ddot{v} & =w,
\end{aligned}
$$

\footnotetext{
${ }^{3}$ In other words, the springs isolate the motors' rotor inertias from the impact dynamics. Since in practice, these would be the rotor inertias reflected through a gear ratio on the order of $30: 1$, removing the rotor inertias from the impact dynamics can result in considerably less energy loss at impacts.
}

where $V$ and $R$ are as in Proposition 1.

Proposition 3: Consider an output $y=h(q, \alpha)$, where $\alpha$ is fixed. Then the following hold:

a) $h$ has uniform vector relative degree 2 for (18) if, and only if, it has uniform vector relative degree 4 for (23);

b) in both cases, the decoupling matrices depend only on $q$ and they are equal, that is $L_{g} L_{f} h=L_{g_{e}} L_{f_{e}}^{3} h$;

c) for $h(q, \alpha)=q_{a}-h_{d}(\theta, \alpha)$,

$$
\operatorname{det}\left(L_{g} L_{f} h\right)(q, \alpha)=1-R\left(q_{a}\right) \frac{\partial h_{d}(\theta, \alpha)}{\partial \theta} ;
$$

d) if $h(q, \alpha)=q_{a}-h_{d}(\theta, \alpha)$ and has uniform vector relative degree 2 for (18), then the zero dynamics manifolds and restriction dynamics of (18) and (23) are diffeomorphic; moreover, the zero dynamics manifold of (18) is

$\mathcal{Z}_{\alpha}=\left\{(q ; \dot{q}) \in T \mathcal{Q} \mid q_{a}=h_{d}(\theta, \alpha), \dot{q}_{a}=\frac{\partial h_{d}(\theta, \alpha)}{\partial \theta} \dot{\theta}\right\}$

and in the coordinates $(\theta ; \sigma)$, the restriction dynamics is

$$
\begin{aligned}
\dot{\sigma} & =\left.\frac{-\partial V}{\partial \theta}\right|_{q_{a}=h_{d}(\theta, \alpha)} \\
\dot{\theta} & =\frac{\sigma}{\tilde{d}_{N, N}(\theta, \alpha)}\left(1-\tilde{R}(\theta, \alpha) \frac{\partial h_{d}(\theta, \alpha)}{\partial \theta}\right)^{-1}
\end{aligned}
$$

where, $\tilde{d}_{N, N}(\theta, \alpha)=\left.d_{N, N}\right|_{q_{a}=h_{d}(\theta, \alpha)}$ and $\tilde{R}(\theta, \alpha)=$ $\left.R\right|_{q_{a}=h_{d}(\theta, \alpha)}$.

Proof: a) and b) are immediate from Propositions 1 and 2. The calculation for c) is a straightforward application of the Sherman-Morrison-Woodbury formula and is left to the reader. The first part of $d$ ) follows from basic results in [12] and equations (28), (29), and (30) follow from [25].

\section{EXAMPLE}

The results of the paper are now illustrated on the 5-link planar biped of Fig. 2, with model given in (24). On the basis of Theorem 2 and Proposition 3, an output function $y\left(x_{e}, \alpha\right)$ and a parameter update function $\Delta_{1}$ will be chosen for (24) so that, with deadbeat parameter updates, it has a valid HZD. Then, using once again Proposition 3 , the conditions of Theorem 1 will be verified, resulting in a feedback $u_{m}\left(x_{e}, \alpha\right)$. The closed-loop system will then be simulated, showing behaviors that are consistent with the theoretical analysis.

\section{A. An Output Function, Feedback, and Update Law}

Motivated by Proposition 3 and [25], the output is selected as

$$
y=h(q, \alpha)=q_{a}-h_{d}\left(\frac{\theta-\theta_{i}}{\theta_{f}-\theta_{i}}, \alpha\right)
$$

where $h_{d}: \mathbb{R} \times \mathcal{A} \rightarrow \mathbb{R}^{4}$ is a $4 \times 1$ vector of Bézier polynomials of degree ${ }^{4} m \geq 7$. The terms $\theta_{i}$ and $\theta_{f}$ are

\footnotetext{
${ }^{4}$ Seventh degree Bézier polynomials have eight independent parameters. This can be shown to be the minimum number of free parameters needed to design the parameter update law and guarantee that $\mathcal{S}_{e} \cap \mathcal{Z}_{e, \alpha}$ is independent
} of $\alpha$. 
constants, equal to the values of $\theta$ at the beginning and end, respectively, of a steady state gait. For any choice ${ }^{5}$ of $\alpha=\left(\alpha_{0}, \alpha_{1}, \alpha_{2}, \alpha_{3}\right) \in \mathcal{A}=\mathbb{R}^{4 \times 4}$, the set of outputs (31) is relative degree 2 with respect to the biped model without actuator dynamics (19), and so by Proposition 3 (a) is relative degree 4 with respect to the biped model with compliant actuation (24). In the context of the model with compliant actuation, differentiating the output four times yields

$$
y^{(4)}=L_{f_{e}}^{4} h\left(x_{e}, \alpha\right)+L_{g_{e}} L_{f_{e}}^{3} h\left(x_{e}, \alpha\right) u_{m},
$$

where the domain of invertibility of the decoupling matrix, $L_{g_{e}} L_{f_{e}}^{3} h\left(x_{e}, \alpha\right)$, is computable using Proposition 3 (b) and (c). The zero dynamics manifold associated with this output is

$\mathcal{Z}_{e, \alpha}=\left\{\begin{array}{l|l}x_{e} \in \mathcal{X}_{e} & \begin{array}{ll}h\left(x_{e}, \alpha\right)=0, & L_{f} h\left(x_{e}, \alpha\right)=0, \\ L_{f}^{2} h\left(x_{e}, \alpha\right)=0, & L_{f}^{3} h\left(x_{e}, \alpha\right)=0\end{array}\end{array}\right\}$,

and is diffeomorphic to the zero dynamics manifold (28). The feedback $u_{m}$ of (10) will render $\mathcal{Z}_{e, \mathcal{A}}:=\cup_{\alpha \in \mathcal{A}}\left(\mathcal{Z}_{e, \alpha}, \alpha\right)$ invariant and exponentially attractive in the continuous phase of the closed-loop system. Note that this feedback is defined using a constant $\epsilon>0$ that is tuned so that $\mathcal{Z}_{e, \mathcal{A}}$ can be made exponentially attractive with arbitrarily fast convergence.

With $h_{d}(\theta, \alpha)$ selected as a Bézier polynomial, it may be shown that $\forall \alpha \in \mathcal{A}, \forall x_{e} \in \Delta_{e}\left(\mathcal{S}_{e} \cap \mathcal{Z}_{e, \diamond}\right)$,

$$
\begin{aligned}
h\left(x_{e}, \alpha\right) & =A_{0}\left(x_{e}\right) \alpha_{0}+B_{0}\left(x_{e}\right) \\
L_{f} h\left(x_{e}, \alpha\right) & =A_{1}\left(x_{e}\right) \alpha_{1}+B_{1}\left(x_{e}, \alpha_{0}\right) \\
L_{f}^{2} h\left(x_{e}, \alpha\right) & =A_{2}\left(x_{e}\right) \alpha_{2}+B_{2}\left(x_{e}, \alpha_{0}, \alpha_{1}\right) \\
L_{f}^{3} h\left(x_{e}, \alpha\right) & =A_{3}\left(x_{e}\right) \alpha_{3}+B_{3}\left(x_{e}, \alpha_{0}, \alpha_{1}, \alpha_{2}\right)
\end{aligned}
$$

with $A_{i}$ 's invertible. This property guarantees that there exists an impact update law, $\Delta_{1}$, satisfying Hypothesis 5 of Theorem 2. Hypotheses 3 and 4 are satisfied by noting that for a five-link biped without impact updated parameters or actuator dynamics, $\mathcal{S} \cap \mathcal{Z}$ is smooth and has dimension one less than $\mathcal{Z}$ itself [25], and that the same derivation applies as long as $\mathcal{S} \cap \mathcal{Z}_{\alpha}$ is independent of $\alpha$. (Such independence has been established earlier by specifying that the last four coefficients of each Bézier polynomial are unaffected by the update law). Hypothesis 1 and 2 have already been established by Proposition 3. Thus, the conditions of Theorem 2 are met and the chosen output $h(q, \alpha)$ will yield a valid HZD of (24) with deadbeat parameter augmentation.

To apply Theorem 1 to the system (14), all that remains is to find an exponentially stable limit cycle within the HZD of (24) and to verify that the coordinate transform of Theorem 1, Hypothesis 2 is valid. This can be done quite efficiently on the basis of the HZD using an optimization technique developed in [24] for finding periodic orbits in the HZD subject to constraints on stability, torque, energy efficiency, ground friction, etc. Using this method, a gait was designed using Matlab's FMINCON function to achieve

\footnotetext{
${ }^{5}$ Note that although the Bézier polynomials of $h(q, \alpha)$ each have 8 coefficients, only the first four components are treated by the parameter update function. The last four must remain constant-after the computation of a periodic orbit-in order that $\mathcal{S}_{e} \cap \mathcal{Z}_{e, \alpha}$ is independent of $\alpha$.
}

a forward progression rate of $0.8 \mathrm{~m} / \mathrm{s}$ and to minimize an approximation of motor electrical energy consumed per distance traveled.

\section{B. Interpreting Simulation Results}

Figure 3 illustrates one interpretation of Theorem 1, namely that while the feedback law $u_{m}\left(x_{e}, \alpha\right)$ of (10) will render $\mathcal{Z}_{e, \mathcal{A}}$ forward invariant and continuous-phase exponentially attractive for any value of $\epsilon>0$, only for $\epsilon$ sufficiently small does it render the manifold exponentially attractive in a hybrid sense. The reason is that for state values outside the zero dynamics manifold, application of the impact map will tend to push the state further away, an effect that can be overcome by sufficiently fast convergence in the continuous phase. This conclusion is reinforced in Fig. 4 , where the spectral radius of the Poincaré return map of the closed-loop system is plotted along with the eigenvalue of the return map associated with the HZD. Figure 5 then shows that the trajectories of the HZD converge to a periodic orbit.

\section{CONCLUSIONS}

Motivated by the problem of creating exponentially stable periodic orbits in bipedal robots with underactuation and actuator dynamics, the hybrid zero dynamics (HZD) framework of [25] has been extended to nonlinear systems with impulse effects where the outputs have vector relative degree higher than two. The formal part of this extension, formulating the proper conditions of simultaneous invariance under the continuous dynamics and the impact map, was straightforward. Even more, an existing result could be used to show that exponentially stable periodic orbits of the HZD can be rendered exponentially stable in the (full-dimensional) closed-loop system with impulse effects. All of this was formalized in Theorem 1.

The more challenging aspect of the extension was to determine how to meet the impact invariance condition when the relative degree is greater than two. The result in [25] on this aspect of the problem could not be extended in a direct way. A novel embedding of the original system into a system with event-based parameter updates was introduced. The additional dynamic elements in the larger system are tailored to meet the boundary conditions associated with impact invariance, and hence the existence of an HZD. This result was formalized in Theorem 2 .

Equipped with Theorems 1 and 2, the motivating problem of creating exponentially stable periodic orbits in a class of planar bipedal robots with underactuation and actuator dynamics was addressed. Key properties of the models were first summarized, in light of applying the main theorems. A set of detailed simulations on a particular example was then provided in order to illustrate and support the prior theoretical developments. 


\section{REFERENCES}

[1] M. Ahmadi and M. Buehler, "The ARL Monopod II running robot: Control and energetics," in IEEE International Conference on Robotics and Automation, May 1999, pp. 1689-1694.

[2] R. Alexander, "Three uses for springs in legged locomotion," International Journal of Robotics Research, vol. 9, no. 2, pp. 53-61, 1990

[3] A. Astolfi and R. Ortega, "Immersion and invariance: A new tool for stabilization and adaptive control of nonlinear systems," IEEE Transactions on Automatic Control, vol. 48, no. 4, pp. 590-606, 2003.

[4] J. G. Cham, S. A. Bailey, J. E. Clark, R. J. Full, and M. R. Cutkosky, "Fast and robust: Hexapedal robots via shape deposition manufacturing," International Journal of Robotics Research, vol. 21, no. 10-11, pp. 869-882, 2002.

[5] V. Chellaboina, S. P. Bhat, and W. M. Haddad, "An invariance principle for nonlinear hybrid and impulsive dynamical systems," Nonlinear Analysis, vol. 53, pp. 527-550, 2003.

[6] J. H. Choi and J. W. Grizzle, "Feedback control of an underactuated planar bipedal robot with impulsive foot action," Robotica, vol. 23, pp. 567-580, September 2005.

[7] _ "Planar bipedal walking with foot rotation," in Proc. of American Control Conference, Portland, OR, June 2005.

[8] J. W. Grizzle, G. Abba, and F. Plestan, "Asymptotically stable walking for biped robots: Analysis via systems with impulse effects," IEEE Transactions on Automatic Control, vol. 46, pp. 51-64, January 2001.

[9] J. W. Grizzle, C. Moog, and C. Chevallereau, "Nonlinear control of mechanical systems with an unactuated cyclic variable," IEEE Transactions on Automatic Control, vol. 30, no. 5, pp. 559-576, May 2005

[10] Y. Hurmuzlu and D. Marghitu, "Rigid body collisions of planar kinematic chains with multiple contact points," International Journal of Robotics Research, vol. 13, no. 1, pp. 82-92, 1994.

[11] J. W. Hurst, J. E. Chestnutt, and A. A. Rizzi, "An actuator with physically variable stiffness for highly dynamic legged locomotion," in Proc. of the 2004 IEEE International Conference on Robotics and Automation, 2004, pp. 4662-4667.

[12] A. Isidori, Nonlinear Control Systems: An Introduction, 3rd ed. Berlin: Springer-Verlag, 1995.

[13] H. Khalil, Nonlinear Systems - 3rd Edition. Upper Saddle River, NJ: Prentice Hall, 2002.

[14] B. Morris and J. W. Grizzle, "A restricted Poincaré map for determining exponentially stable periodic orbits in systems with impulse effects: Application to bipedal robots," in Proc. of IEEE 2005 Conference on Decision and Control, 2005.

[15] S. Nersesov, V. Chellaboina, and W. Haddad, "A generalization of Poincaré's theorem to hybrid and impulsive dynamical systems," International Journal of Hybrid Systems, vol. 2, pp. 35-51, 2002.

[16] I. Poulakakis, J. A. Smith, and M. Buehler, "Modeling and experiments of untethered quadrupedal running with a bounding gait: The Scout II robot," International Journal of Robotics Research, vol. 24, no. 4, pp. 239-256, 2005.

[17] J. Pratt, B. Krupp, and C. Morse, "Series elastic actuators for high fidelity force control," Industrial Robot Journal, vol. 29, no. 3, pp. 234-241, 2002.

[18] J. E. Pratt and B. T. Krupp, "Series elastic actuators for legged robots," SPIE, Unmanned Ground Vehicle Technology VI, vol. 5422, no. 1, pp. 135-144, 2004.

[19] M. Reyhanoglu, A. van der Schaft, N. McClamroch, and I. Kolmanovsky, "Dynamics and control of a class of underactuated mechanical systems," IEEE Transactions on Automatic Control, vol. 44, no. 9, pp. 1663-1671, 1999.

[20] U. Saranli, M. Buehler, and D. Koditschek, "RHex: A simple and highly mobile hexapod robot," International Journal of Robotics Research, vol. 20, no. 7, pp. 616-631, July 2001.

[21] M. Spong, "Modeling and control of elastic joint robots," Journal of Dynamic Systems, Measurement, and Control, vol. 109, pp. 310-319, Dec. 1987.

[22] — " "Adaptive control of flexible joint manipulators," Systems \& Control Letters, vol. 13, pp. 15-21, 1989.

[23] — - "Energy based control of a class of underactuated mechanical systems," in Proc. of IFAC World Congress, San Francisco, CA, 1996, pp. $431-435$.

[24] E. R. Westervelt and J. W. Grizzle, "Design of asymptotically stable walking for a 5-link planar biped walker via optimization," in Proc. of the 2002 IEEE International Conference on Robotics and Automation, Washington, D.C., 2002, pp. 3117-3122.
[25] E. R. Westervelt, J. W. Grizzle, and D. E. Koditschek, "Hybrid zero dynamics of planar biped walkers," IEEE Transactions on Automatic Control, vol. 48, no. 1, pp. 42-56, January 2003.

[26] H. Ye, A. Michel, and L. Hou, "Stability theory for hybrid dynamical systems," IEEE Transactions on Automatic Control, vol. 43, no. 4, pp 461-474, April 1998
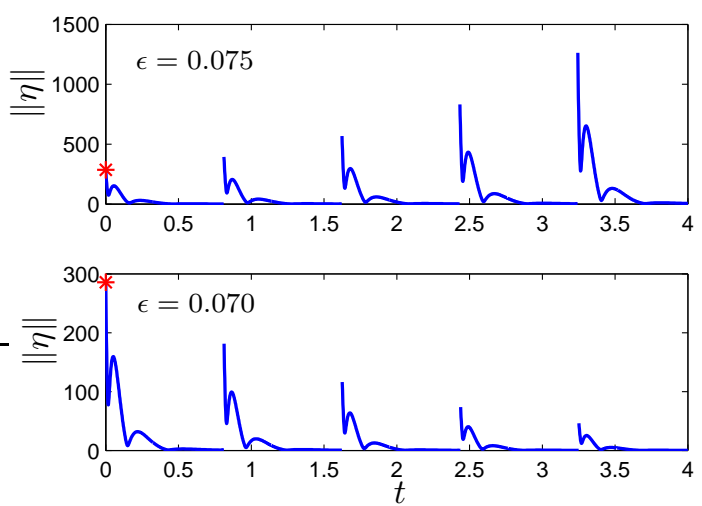

Fig. 3. The above plot shows that under the feedback (10), for a particular choice of gain matrices, $K_{i}$, the zero dynamics manifold is attractive for $\epsilon=0.070$ but not for $\epsilon=0.075$. Plotted on the vertical axis is the euclidian norm of $\eta=\left(h(q, \alpha) ; L_{f_{e}} h\left(x_{e}, \alpha\right) ; L_{f_{e}}^{2} h\left(x_{e}, \alpha\right) ; L_{f_{e}}^{3} h\left(x_{e}, \alpha\right)\right)$. The horizontal axis is time. The observed behavior is consistent with Theorem 1 , where the zero dynamics manifold $y \equiv 0$ is made exponentially attractive for sufficiently small $\epsilon$ with the feedback (10). Initial conditions for the two plots are the same and indicated by an asterisk.

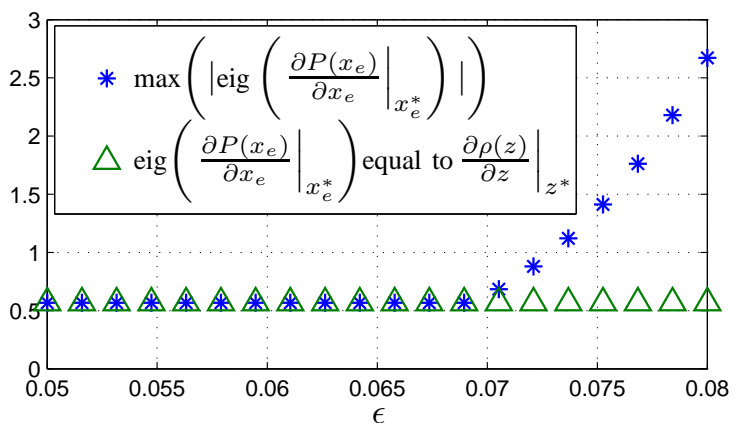

Fig. 4. The above plot shows how the eigenvalues of the linearization of the return map vary with the parameter $\epsilon$ of the feedback (10). As $\epsilon$ approaches zero, one eigenvalue remains constant, equal to the eigenvalue of the hybrid zero dynamics $\partial \rho(z) / \partial z$, while all other eigenvalues go to zero. The eigenvalue associated with the 1DOF HZD is 0.567 .

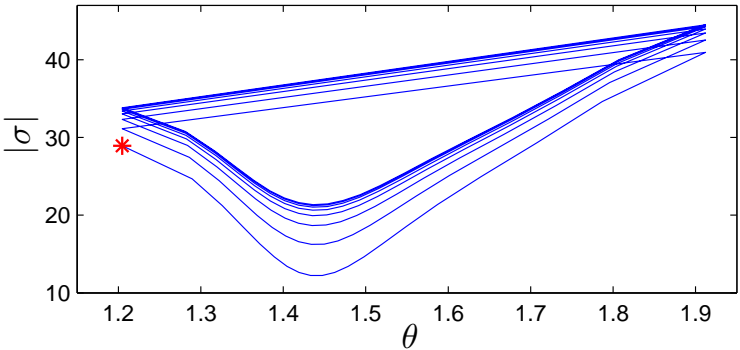

Fig. 5. Theorem 1 links the stability of an orbit within the hybrid zero dynamics to the stability of an orbit in the full system. The above shows the system response to an initial condition (noted with an asterisk) within the hybrid zero dynamics but not on the periodic orbit. The state converges exponentially quickly back to the periodic orbit. 